\begin{tabular}{|c|l|}
\hline Title & Reliable phase unwrapping algorithm based on rotational and direct compensators \\
\hline Author(s) & Heshmat, Samia; Tomioka, Satoshi; Nishiyama, Shusuke \\
\hline Citation & $\begin{array}{l}\text { A pplied Optics, 50(33), 6225-6233 } \\
\text { https://doi.org/10.1364/A0.50.006225 }\end{array}$ \\
\hline Issue Date & 2011-11-20 \\
\hline Doc URL & http://hdl.handle.net/2115/48197 \\
\hline Rights & ○ 2011 Optical Society of A merica \\
\hline Type & article \\
\hline File Information & A 050-33_6225-6233.pdf \\
\hline
\end{tabular}

Instructions for use 


\title{
Reliable phase unwrapping algorithm based on rotational and direct compensators
}

\author{
Samia Heshmat, ${ }^{1,2, \star}$ Satoshi Tomioka, ${ }^{3}$ and Shusuke Nishiyama ${ }^{3}$ \\ ${ }^{1}$ Graduate School of Engineering, Hokkaido University, Kita 13 Nishi 8, Kita-ku, Sapporo, Hokkaido 060-8628, Japan \\ ${ }^{2}$ Faculty of Engineering, South Valley University, Aswan 81542, Egypt \\ ${ }^{3}$ Faculty of Engineering, Hokkaido University, Kita 13 Nishi 8, Kita-ku, Sapporo, Hokkaido 060-8628, Japan \\ ${ }^{*}$ Corresponding author: samia @ athena.qe.eng.hokudai.ac.jp
}

Received 5 August 2011; revised 2 October 2011; accepted 3 October 2011; posted 5 October 2011 (Doc. ID 152448); published 16 November 2011

\begin{abstract}
Phase unwrapping still plays an important role in many data-processing chains based on phase information. Here, we introduce a new phase unwrapping approach for noisy wrapped phase maps of continuous objects to improve the accuracy and computational time requirements of phase unwrapping using a rotational compensator (RC) method. The proposed algorithm is based on compensating the singularity of discontinuity sources. It uses direct compensation for adjoining singular point (SP) pairs and uses RC for other SP pairs. The performance of the proposed method is tested through both simulated and real wrapped phase data. The proposed algorithm is faster than the original algorithm with the RC and has proved efficiency compared to other phase unwrapping methods. (C) 2011 Optical Society of America OCIS codes: $\quad 030.4280,100.3175,100.5088,110.5086,120.5050$.
\end{abstract}

\section{Introduction}

Two-dimensional (2D) phase unwrapping is key to data-processing chains in many fields of research, such as optics [1], magnetic resonance imaging [2,3], and synthetic aperture radar interferometry $[4,5]$. In all these research fields, the measured parameters are modulated in the form of $2 \mathrm{D}$ fringe pattern. To retrieve the phase information from the fringe pattern, Fourier domain filtering [6-8] or phase shift technique $[\overline{7}, \underline{9}, 10]$ can be used. The retrieved phase values, which are wrapped phase, are the distribution of principal values ranging from $-\pi$ to $\pi$. Thus, phase unwrapping procedure is needed to get back the unknown multiple of $2 \pi$ to each pixel. However, the defects in the fringe patterns, such as phase discontinuity, shadow, and/or noise, are the main difficulties in the phase unwrapping methods. To exclude these invalid areas from the unwrapping process and obtain precise unwrapped phase results can be a time-consuming process.

0003-6935/11/336225-09\$15.00/0

(C) 2011 Optical Society of America
Most phase unwrapping algorithms are divided into two basic classes. The first class is algorithms based on following the paths [4,11-16]. In these methods, the unwrapping operation is carried out along paths of successive pixels, where the paths are taken to avoid inconsistent pixels. These inconsistencies arise from the existence of discontinuity sources. The path-following methods are fast, however, they may contain unintended gaps of unwrapped phase in the case where phase data contains high ratio of error sources, such as noise and phase discontinuities of more than $\pi$. This is a serious problem for quantitative evaluation of $2 \mathrm{D}$ optical information. The second class of algorithms is based on a least-squares approach [1,17-22]. These methods use a principle that minimizes the differences between the partial derivatives of wrapped phase differences and the partial derivatives of the solution (unwrapped phase differences). Phase unwrapping using the rotational compensator (RC) algorithm [23] and singularity-spreading phase unwrapping (SSPU) method [24] are also classified into the same class of least-squares methods, since they 
spread the singularity similar to the least-squares methods. For noisy data, these methods provide accurate unwrapped results. For example, the RC algorithm [23] is the most accurate method among Goldstein's path following [4], the least-squares method with discrete cosine transform (LS-DCT) [22], and SSPU [24] methods. It can compensate the singularities of singular points (SPs). Although the effect of compensation decreases with the increasing distance from the SP, it does not perfectly vanish in a distant area from SP, even if the area has no SPs. This is considered an error in phase unwrapping by RC. Furthermore, it requires large amount of computational cost.

The phase unwrapping methods mentioned earlier deal with the noise problem in several different ways. The path-following methods suppress the noise by laying branch cuts to avoid residues. On the other hand, the least-squares methods fit the phase surface to provide the solution. However, there is another type of method that performs phase map denoising before phase unwrapping. For this method, efforts have been made over the last decades to remove noise (denoising) before phase unwrapping. A wrapped phase maps can be easily unwrapped by filtering the noise properly. However, it is difficult to filter the wrapped phase. To reduce the phase map noise, several methods have been proposed, such as the multilook filter method [25], the local statistic filter, and Fourier transform-based methods [26,27]. Although these techniques can reduce the noise within the original spatial resolution, they are highly dependent on the window size. The phase data is considered as a nonstationary signal [28], and the Fourier transform cannot process nonstationary signals. For those reasons, Goldstein and Werner proposed a filter dividing the phase data into small blocks in which the spectrum of each block is filtered separately [26]. The windowed Fourier transform (WFT) and wavelet transform are regarded as two spatial frequency analyses having the ability of processing signals locally. Recently, in order to reduce the phase noise based on WFT, a novel algorithm has been proposed [29-31]. This algorithm uses a constant threshold in different cases. This could be considered a deficiency for this algorithm. Moreover, a proper window size is critical for yielding good filtering results. Choosing a small window size will reduce the linear phase approximation errors. On the other hand, choosing a large window size will reduce the influence of the noise. Hence, the window size should be selected to take the balance between the linear phase approximation error and the noise level. Inadequate choice of window size will affect phase retrieval.

Based on the above discussion, the existing phase unwrapping methods suffer from various problems that can affect time, cost, and accuracy of unwrapped results. Therefore, it is needed to investigate and improve the existing unwrapping methods. In this paper, we attempt to reduce the computational time requirements of the $\mathrm{RC}$ method to a minimum, and to improve the level of efficiency and reliability as well. It was found that the distribution of dipole distance shows that there are a lot of dipole pairs that have short distances. According to this finding, the proposed algorithm is computing compensators for adjoining pairs of SPs directly; the new method is a coupling of the $\mathrm{RC}$ and the direct compensator (DC). The remainder of this paper is organized as follows: the explanation and analysis of phase unwrapping is shown in Section 2 , while Section 3 presents the basic idea and procedures of the proposed method. Section 4 demonstrates the applicability and validity of the proposed algorithm by comparing its unwrapped results with the past methods using simulation and experimental data. Finally, the conclusion is given in Section 5 .

\section{Analysis of Phase Unwrapping}

\section{A. Phase Unwrapping Problem}

Commonly, most phase unwrapping algorithms are based on one assumption: that the true unwrapped phase data, $\Phi_{i}$, varies gradually enough to make the neighboring phase difference values be within one half cycle $(\pi \mathrm{rad})$ of each other, $\left|\Delta \Phi^{i}\right|=$ $\left|\Phi_{i+1}-\Phi_{i}\right|<\pi$. If this assumption is true everywhere, the unwrapping process can be applied simply by integrating wrapped phase differences, or gradients, along any path from a pixel to another pixel throughout the phase data to obtain an unwrapped phase. The wrapped gradient phase difference, $\hat{\nabla} \Psi^{i}$, between two successive pixels in the unwrapping path can be defined as

$$
\begin{aligned}
\hat{\nabla} \Psi^{i} & =\Delta \Psi^{i}-\operatorname{Int}\left[\frac{\Delta \Psi^{i}}{2 \pi}\right] 2 \pi, \\
\Delta \Psi^{i} & =\Psi_{i+1}-\Psi_{i}
\end{aligned}
$$

where $\Psi_{i}$ is the wrapped phase at pixel $i$ in the phase map, and Int[] means a function that returns the nearest integer. The absolute wrapped gradient, $|\hat{\nabla} \Psi i|$, is always smaller than $\pi$. In the absence of discontinuity sources, the unwrapped result is independent of the unwrapping path; therefore, the unwrapped phase map is consistent. Considering that a path consists of $M$ points, the points are numbered from 0 to $M-1$. If the difference of the true unwrapped phases satisfies this relation $\left|\Delta \Phi^{i}\right|<\pi$, since the wrapped gradient is identical to the difference of the true phases, $\hat{\nabla} \Psi^{i}=\Delta \Phi^{i}$, we can retrieve the true unwrapped phase as follows:

$$
\Phi_{M}=\Phi_{0}+\sum_{i=0}^{M-1} \hat{\nabla} \Psi^{i} .
$$

In $2 \mathrm{D}$ phase unwrapping, there are paths with a loop, which means that the last point can be considered the first point. In the case where point $M$ 
is identical to point 0 , if the relation $\left|\Delta \Phi^{i}\right|<\pi$ is satisfied, the summation of $\nabla \Psi^{i}$ for all points (from 0 to $M-1$ ) in the path must equal zero. However, in the presence of discontinuities, the path of integration becomes dependent and choosing a path randomly is not possible anymore. If Eq. (2) is used by itself to retrieve the unwrapped phase map, it may result in the addition or the subtraction of incorrect multiples of $2 \pi$, which will then propagate throughout the rest of the phase map. Restrictions must be applied to the unwrapping path in the corrupted areas, which result in the path being dependent. To avoid this situation, corrupted areas, or SPs, must be identified, balanced, and isolated from the rest of the nonsingular pixels using barriers (branch cuts) in the phase map. Once SPs are isolated, phase unwrapping will take an independent path avoiding these branch cuts, therefore, it retrieves the true phase.

\section{B. SPs and Branch Cuts}

A path in the $2 \mathrm{D}$ phase map consists of a sequence of horizontal and vertical segments joined at their adjacent points. To calculate SPs, it was applied that a closed path with four segments $(i=0, \ldots, 3)$ starting in every point defined by the corners of a $2 \times 2$ square. The SPs are identified by summing $\hat{\nabla} \Psi^{i}$ along the closed path in clockwise direction, as follows:

$$
\sum_{i=0}^{3} \hat{\nabla} \Psi^{i}=2 \pi S,
$$

where $S$ is the residue of SP. When the value of $S$ in Eq. (3) is +1 or -1 , SP can be found. The SP is called a positive residue when $S$ is +1 ; otherwise, it is called a negative residue when $S$ is -1 .

When closed path includes the same number of SPs with positive residues and those with negative residues, the integral along the chosen path is equal to zero. In this way, the unwrapping process is carried out successfully outside the closed path. In the area inside the closed path, lines between SPs with different signed residues are placed, which are called branch cuts. These branch cuts act as barriers to prevent the unwrapping path to cross them [11-16]. Basically, SPs appear as pairs of poles with the opposite sign. However, some isolated SPs or SPs of dipoles with long distances, which are called monopoles, may appear near the boundaries of the phase map because the measurement domain is finite. This can result in the number of positive SPs and negative ones in the measurement area being different. Moreover, monopoles spread errors throughout the entire measurement area [23]. Therefore, it is necessary to balance the number of positive and negative SPs. One solution is to append virtual SPs (VSPs) outside the measurement area, so that the error of monopoles will be reduced. There are several positive and negative SPs in the phase map, hence, there will be numerous possible ways to link them. For any possible set of links, the unwrapped phase map is not the same, despite this, only one unwrapped phase map is correct. To find the correct set of linkages, a criterion has to be set as an evaluation for the quality of the unwrapped result. One way for coupling SPs and forming dipole pairs is the unconstrained SP (USP) positioning technique [23]. Generally, the SP is positioned at the center point of an elementary path that consists of four adjacent pixels with square shape. When the SPs are located at the center of the loop, it may be difficult to determine which SPs are the closest couple to each other, since the distances between the SPs are distributed in discrete values and some SPs may be located at the same distance. However, if USP technique is used, we can obtain the accurate positioning of the SPs, which means that their positions are obtained in a continuous manner. Hence, the distances are different in most cases, and we can determine which SPs are the closest to each other to form a pair.

\section{Phase Unwrapping by RC and DC Algorithms}

In a manner similar to the phase unwrapping algorithm developed by Tomioka et al. [23], the main issues determine the behavior of the proposed algorithm: the RC, USP positioning, and VSP approach to compensate the inconsistencies and to confine the effect of each one in a local region. The proposed algorithm is based on their method, however, the way of computing the compensators for adjoining SP pairs is different from RC. The following subsection explain and discuss the $\mathrm{RC}$ and $\mathrm{DC}$ principles and the description of the proposed algorithm.

A. $\mathrm{RC}$

Since phase unwrapping is an essential process of removing discontinuities by local neighborhood tests and corrections, the idea of compensator is proposed to compensate and cancel the singularity effect by the SSPU [24] or RC methods [23]. The SSPU method requires iteration process to compute the compensators, while the RC method can compute the compensator by superposing the effect of each SP. RC can cancel singularity of each SP by adding an integral of isotropic singular function along any loops. When a closed loop includes SP, the integral along the loop will have a value of $-2 \pi S$, where $S$ is the residue of the SP shown in Eq. (3). Representing an integral of a segment $i$, which is a member of the loop comprising $N$ segments, as $C^{i}$, we can reduce Eq. (3) to

$$
\sum_{i=0}^{N-1}\left(\hat{\nabla} \Psi^{i}+C^{i}\right)=0
$$

This suggests that the singularity of $\Psi^{i}$ is regularized by compensator $C^{i}$, and phase unwrapping becomes an independent path. The RC for the $i$ th segment, which is a path from $\mathbf{r}_{i}$ to $\mathbf{r}_{i+1}$ to cancel the singularity of the $j$ th $\mathrm{SP},{ }^{R} C_{j}^{i}$, is represented as follows: 


$$
{ }^{R} C_{j}^{i}=-S_{j}\left(\theta_{i+1, j}-\theta_{i, j}\right),
$$

where $S_{j}$ denotes the residue of the $j$ th SP, and $\theta_{i+1, j}$ and $\theta_{i, j}$ are azimuthal angles of both ends of the $i$ th segment, where the origin is located at the $j$ th SP.

When the measured data contains several SPs, the total compensator of the $i$ th segment is estimated as the summation of the ${ }^{R} C_{j}^{i}$ with respect to $j$ :

$$
{ }^{R} C^{i}=\sum_{j=1}^{N_{s}}{ }^{R} C_{j}^{i} .
$$

Consequently, we can retrieve the true unwrapped phase data by summing the phase differences between the adjoining pixels and the total compensators as follows:

$$
\Phi_{M}=\Phi_{0}+\sum_{i=0}^{M-1}\left(\hat{\nabla} \Psi^{i}+C^{i}\right)
$$

where $C^{i}={ }^{R} C_{j}^{i}$ and the path is composed from 0 to $M-1$ segment.

It is noteworthy that Eq. (7) is the modification of Eq. (2) after removing the effect of SPs by compensating each SP with the compensator, which has the opposite sign of SP. However, if the measured phase data contains several SPs, the computations of each compensator becomes a time-consuming process. This is one of the drawbacks of the RC method.

Despite the RC, which can remove the inconsistencies in the phase map by eliminating the effect of singularity, it introduces an undesirable distortion of phase in a wide area. The effect of RC of the $j$ th SP, ${ }^{R} C_{i}^{i}$, decreases by the increases the distance from that SP. It becomes small for the distant segment, however, it is not exactly zero. This means that RC affects the regular region, and its effect is considered as an error of phase unwrapping. In the literature [23], the effect of single SP, monopole SP, is proportional to the reciprocal of the distance $R$ from the residue:

$$
\left|{ }^{R} C_{j}^{i}\right| \propto \frac{1}{R}
$$

In contrast, the phase unwrapping method based on avoiding branch cuts does not affect the distant segment at all. In other words, the branch cut approach can precisely confine the singularity of SP pairs within local region, but the RC method spreads the effect of singularity to the whole region. This is another drawback of RC method.

\section{B. DC}

To overcome the drawbacks of RC method, which are high cost of computational time and undesired phase errors due to its effect on regular region, we propose a new algorithm. The proposed algorithm offers simple computations to compensate for the inconsistencies caused by the pairs of the adjoining SPs by adding a DC, so, the effect of each SP is confined within a closer local region. As a result, the drawbacks of the $\mathrm{RC}$ method can be improved. $\mathrm{RC}$ along a segment is defined by computing the summation of the differences between the azimuthal angles for the end points of the segment for all SPs, in which each SP is located at the origin, as illustrated in Eqs. () and (6). Hence, the summation value of $\mathrm{RC}$ along all segments of $2 \times 2$ square loop should be equal to the summation of $\hat{\nabla} \Psi^{i}$ along the path with the opposite sign, as shown in Eq. (4). In the DC case, the compensator value along the segment that crosses the branch cut of the adjoining pair equals the value of the phase jump, which is one cycle $(2 \pi \mathrm{rad})$.

An adjoining pair is a dipole, which consists of two SPs with the opposite sign separated by one pixel horizontally or vertically. Figure 1 shows the configuration of the branch cuts placed between adjoining SPs in the phase map and the concept of the direct compensation; for simplicity, the SPs positions are defined at the center of the closed loops. In Fig. 1, the thick arrows across the branch cuts, which are shown as thick dashed lines, represent the positions of DCs. Figure 1(a) shows a case in which the branch cut is placed between a pair of adjoining SPs horizontally, so that the DC will be added to the vertical segment that crosses the branch cut. The compensator value of the segment is divided into two compensator values and distributed through the two adjacent loops that contain the adjoining SPs, as illustrated in Fig. 1(b). The following equation explains the $\mathrm{DC}$ of a segment that's related to the adjoining pair:

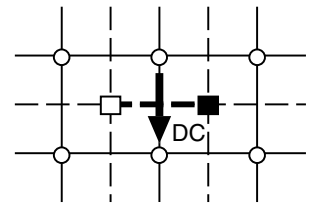

(a)

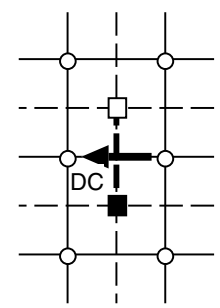

(c)

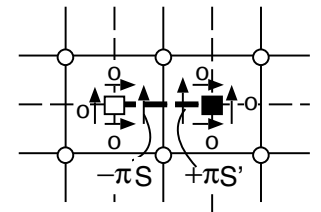

(b)

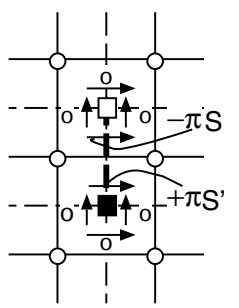

(d)
Fig. 1. Existence of the branch cuts between the adjoining SPs and the concept of direct compensation. Open and filled squares represent positive and negative SPs, respectively. The thick dashed line denotes the branch cut that connects two SPs of opposite signs. Compensator position is denoted by thick arrows. The thin arrows show the direction and distribution of compensators for the segments of each SP, where $S$ and $S^{\prime}$ denote the residues of the SPs. 


$$
{ }^{D} C_{j}^{i}= \begin{cases}T_{j}^{i} \pi S_{j} & \begin{array}{l}
\text { when the segment number } i \\
\text { is a member of the loop of } \\
\text { the } j \text {-th SP, which belongs } \\
\text { to the adjoining pair; } \\
\text { otherwise; }
\end{array}\end{cases}
$$

where $T_{j}^{i}$ denotes the sign direction of ${ }^{D} C_{j}^{i}$, and $S_{j}$ denotes the residue of the $j$ th SP. When the DC of the segment is added, the sign direction of DC for this segment, $T_{j}^{i}$, is dependent on the position of this segment with respect to the location of the tested SPs. For example, when a vertical segment is on the righthand of the tested SP, the sign of $T_{j}^{i}$ is negative (-), and vice versa, as shown in Fig. 1(b). On the other hand, Fig. 1(c) shows the case in which the branch cut is placed vertically between the adjoining SPs and the DC is added horizontally. Thus, when the segment is above the tested SP, the sign of $T_{j}^{i}$ is positive (+), and vice versa, as shown in Fig. 1(d). Also, it is important to discuss the complex distribution of SPs positioning patterns and how we compensate the singularities of these SPs. Figure 2 represents an example of the distribution with four SPs in the phase map. If we consider the distribution of these SPs in a discrete values, the distance between each other will be the same, as shown in Fig. 2(a). Therefore, it is difficult to determine which SPs are the closest couple to each other to form a pair. However, if we use USP method [23] to obtain an accurate positioning of each SP, as shown in Fig. 2(b), we can pick a SP and another one nearby to form a pair. Therefore, the distance between the two SPs of each pair will be defined precisely and the type of pair will be distinguished without difficulty. Thus, DC is added to the adjoining pairs, as illustrated in Fig. 2(b).

Since the DC affects just the brunch cut, the effect does not propagate to the regular region. Moreover, the DC of a segment $i$ for all SPs, ${ }^{D} C^{i}$, needs only two computations in contrast to the RC for another segment $i,{ }^{R} C^{i}$, which needs multiple computations to evaluate effects of all SPs, according to Eq. (6). Therefore, the computational time requirements of the proposed algorithm for computing total compensators

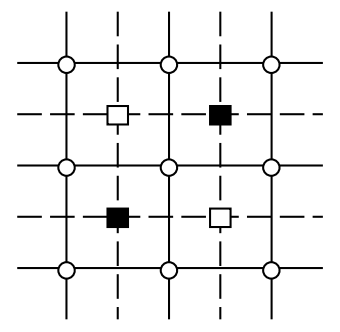

(a)

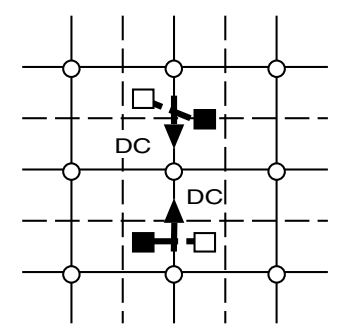

(b)
Fig. 2. Complex cases for the position patterns of SP pairs and the DCs for the adjoining SPs pairs. Open and filled squares represent positive and negative SPs, respectively. (a) SPs are distributed in discrete values. (b) SPs are distributed by using the USP technique. In (b), the thick dashed line denotes the branch cut that connects two SPs of opposite signs, and the DC positions are denoted by thick arrows. will be reduced and the accuracy of the unwrapped phase will be improved, as discussed in Section 4.

The proposed method is based on coupling the $\overline{\mathrm{R}} \mathrm{C}$ and the DC to compute the compensators depending on the converging distance of SPs. In other words, it uses DC for computing the compensators of the pairs of adjoining SPs, and uses RC to compute the compensators for the other pairs. To explain that, it is assumed that new parameters $\operatorname{adj}^{+}(i)$ and $\operatorname{adj}^{-}(i)$, called adjoining parameters of the segment, which is a member of the closed path of the adjoining SPs pair. The $\operatorname{adj}^{+}(i)$ and $\operatorname{adj}^{-}(i)$ denote the positive SP number and the negative SP number, which belongs to the adjoining pair, respectively, where $i$ represents the segment number. It means when the segment $i$ is a joint between the loop of the positive SP and that of the negative SP of the adjoining pair, these parameters have values; otherwise, they are undefined. Considering another parameter $m_{j}^{i}$, which is defined, $m_{j}^{i}=1$ when $\operatorname{adj}^{+}(i)$ or adj- $(i)$ has a value of $j$; otherwise $m_{j}^{i}=0$. It is like Kroneckor's delta function as follows:

$$
m_{j}^{i}=\delta_{j}^{\mathrm{adj}+(i)}+\delta_{j}^{\mathrm{adj}-(i)} .
$$

Therefore, the total compensators can be estimated as follows:

$$
C^{i}=\sum_{j=1}^{N_{s}} \bar{m}_{j}^{i} \cdot{ }^{R} C_{j}^{i}+\sum_{j=1}^{N_{s}} m_{j}^{i} \cdot{ }^{D} C_{j}^{i},
$$

where $\bar{m}_{j}^{i}=1-m_{j}^{i}$. When $\bar{m}_{j}^{i}=1$, i.e., the $\operatorname{SP} j$ does not belong the adjoining pair, it represents the case of using RC to compute the compensator for the segment number $i$ of the $j$ th SP. It should be noted that the times number of $m_{j}^{i} \neq 0$ in the second summation is once for all $i$ for each $j$ related to the adjoining SPs pairs. By adding the compensator, $C^{i}$, to the wrapped phase differences according to Eq. (7), phase jumps were found because the existence of SPs are canceled, therefore, the unwrapped phase can be retrieved successively.

\section{Description of the Algorithm}

The steps of the proposed algorithm can be summarized as follows.

1. Calculate SPs in the wrapped phase map by the summation of the phase gradients of a $2 \times 2$ closed loop path. The positions of SPs are determined by the USP technique.

2. Appending VSPs to the monopole SPs outside the measurement area, analyze the SP pairs, which consist of two real SPs laid inside the measurement area or one real SP and one VSP appended outside the measurement area. After that, define the adjoining SPs pairs.

3. For each segment $i$, the parameter $m_{j}^{i}$ for every SP $j$ is evaluated. If $m_{j}^{i}=1$, i.e., a pair of SPs is an adjoining pair, a DC will be added, according to 
Eq. (9). Otherwise, RC will be computed using Eq. (5).

4. Compute the total compensator for each segment through the whole phase map according to Eq. (11).

5. Finally, the unwrapped phase data can be retrieved by adding the compensators to the wrapped phase differences by using Eq. (7).

This description of direct compensation for the pairs of adjoining SPs makes the proposed algorithm simple and easy to implement. It provides a fast and efficient way to unwrap the phase map. In Section 4, the performance and applicability of the proposed algorithm is examined.

\section{Results}

To evaluate the performance of the proposed phase unwrapping algorithm, both simulated and real wrapped phase maps have been used. These phase data are the same data that were used in the study of $\mathrm{RC}$ [23].

\section{A. Computer Simulation Results}

In order to demonstrate the applicability of the proposed approach, a simulated noisy phase map with constant gradient is generated. This phase data has the image size 100 pixels $\times 100$ pixels, the gradient is $(0.1,-0.1)$ cycle/pixel, and the noise has a normal distribution with 0.15 cycle standard deviation. The original and wrapped phase data are shown in Figs. $3(\mathrm{a})$ and $3(\mathrm{~b})$, respectively. In addition, Fig. 3 presents the distribution patterns of SP pairs for real and virtual SPs to show the position of SP pairs in the phase map. In Fig. 3(c), all SP pair positions are presented, while in Figs. 3(d) and 3(e), the positions of the pairs of nonadjoining and adjoining SPs are shown, respectively. This indicates that most of SP pairs in the phase map are adjoining pairs, therefore, the use of DC will have an obvious effect on the unwrapping process. Hence, the accuracy of the unwrapped phase will be improved and the computation time will be reduced, as shown later. The unwrapped phase results obtained by the LS-DCT method [22], the RC method [23], and the proposed algorithm are shown in Figs. $\overline{3(\mathrm{f})}$ and 3(h) with contour lines. To evaluate the characteristics of the phase unwrapping methods, we can count the number of contour lines in the unwrapped results and compare them with the number of stripes in the wrapped data, shown in Fig. 3(b). From the comparison, we can find that the number of lines in the unwrapped results is less than that in the wrapped phase data. The wrapped phase data has 20 lines, the unwrapped result of LS-DCT method has 14 lines, the unwrapped result of $\mathrm{RC}$ algorithm has 17 lines, and the proposed algorithm's result has 18 lines. The unwrapped result of the proposed algorithm has the nearest number of lines to wrapped data, which shows the highest accuracy. Moreover, the accuracy of the proposed algorithm can

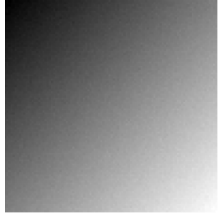

(a)

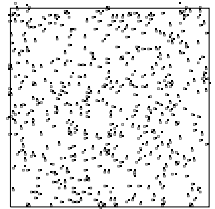

(c)

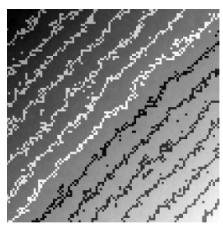

(f)

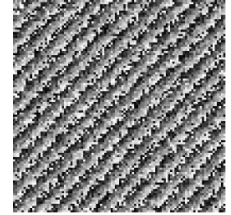

(b)

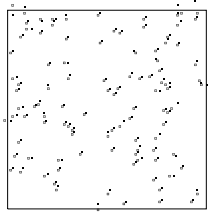

(d)

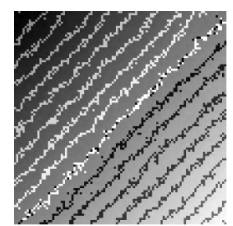

(g)

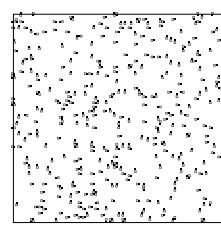

(e)

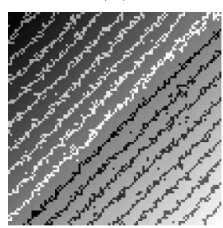

(h)
Fig. 3. Comparison of the unwrapped phase results for simulated phase data: (a) original phase data, (b) wrapped data, (c) positions of all SP pairs, (d) positions of the pairs of nonadjoining SPs, (e) positions of the pairs of adjoining SPs, (f) unwrapped result by LS-DCT, (g) unwrapped result by RC, and (h) unwrapped result by RC + DC (proposed). In (a), (b), and (f)-(h), the phase increases with the increases of brightness. In (f)-(h), contour lines of the phase with the interval of one cycle are also shown.

be emphasized, as shown in Table 1 , which shows a quantitative comparison of the original and unwrapped phase map gradients. The gradients are obtained by fitting them to a planar function, $\tilde{\phi}$, and the $\sigma$ denotes the mean residual that is defined as a square root of a mean square residual from the fitted function, $\tilde{\phi}$. The $\sigma$ of the original phase data is not equal to zero, because the original data contains noise with the given standard deviation. The errors of gradient, $\Delta(\nabla \tilde{\phi})$, is estimated as the normalized difference between the unwrapped result and the original one, where the normalizing factor is the reciprocal of original one. From the table, it can be observed that the proposed algorithm, RC $+\mathrm{DC}$, gives the smallest error in terms of $\Delta(\nabla \tilde{\phi})$. This is due to the consideration of adjoining pair definition in computation of the compensators in the proposed algorithm, as explained in Section 3. This result confirms that the proposed method $(\overline{\mathrm{R}} \mathrm{C}+\mathrm{DC})$ reduces

Table 1. Comparison of the Accuracy for the Simulation Data Shown in Fig. $\underline{3}$

\begin{tabular}{|c|c|c|c|c|}
\hline & Algorithm & $\begin{array}{c}\text { Gradient }(\nabla \tilde{\phi}) \\
\quad(\text { cycle/pixel })\end{array}$ & $\Delta(\nabla \tilde{\phi})(\%)$ & $\sigma($ cycle/pixel $)$ \\
\hline & Original & $(0.1000,-0.1000)$ & $(-,-)$ & 0.149 \\
\hline (a) & LS-DCT & $(0.0742,-0.0731)$ & $(-25.8,-27.0)$ & 0.179 \\
\hline (b) & $\mathrm{RC}$ & $(0.0912,-0.0896)$ & $(-8.7,-10.4)$ & 0.168 \\
\hline (c) & $\mathrm{RC}+\mathrm{DC}$ & $(0.0956,-0.0951)$ & $(-4.4,-4.9)$ & 0.168 \\
\hline
\end{tabular}




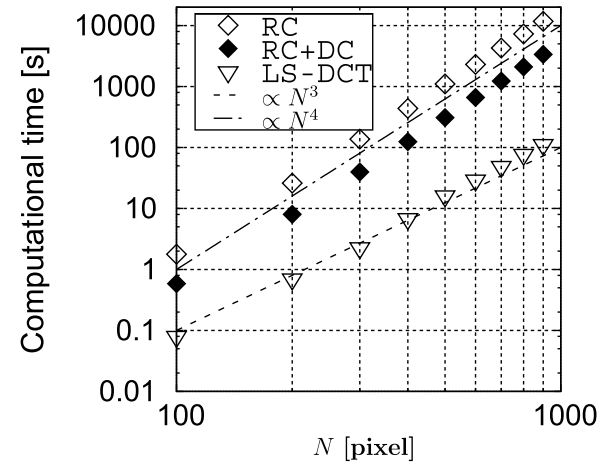

Fig. 4. Required computational time of each algorithm for various image sizes. The horizontal axis $N$ denotes one-dimensional area size in pixels. $\mathrm{RC}$ shows the required time cost for $\mathrm{RC}$ method, $\mathrm{RC}+\mathrm{DC}$ shows the required execution time for the proposed method, and LS-DCT shows the required time cost for LS-DCT method. The computational time is measured with a PC including an Intel Core 2 DUO central processing unit (CPU) with a $2.13 \mathrm{GHz}$ clock in the single CPU operation mode.

the phase errors that exist mainly in the original $\mathrm{RC}$ method.

Figure 4 shows a comparison of required computational time of LS-DCT and RC methods and RC + DC (the proposed method) for various image sizes; the horizontal axis $N$ denotes one-dimensional area size in pixels. From the figure, the profile of the RC method and that of the proposed method show that the computation time is proportional to $N^{4}$. Furthermore, from Eq. (6), we can note that the time cost to compute the $\overline{\mathrm{RC}}$ for all segments is proportional to the product of both the number of SPs and the number of the segments of path to be compensated. Since both are proportional to the area size $\left(\propto N^{2}\right)$, the total evaluation time is proportional to $N^{4}$. In the proposed algorithm, if the cost to compute the $\mathrm{DC}$ is adequately smaller than that of $\mathrm{RC}$, the total cost might be similar to the case of the RC algorithm, and it can be understood from Eq. (11). Conversely, when the number of the times using $\overline{\mathrm{DC}}$ computation is larger than that of $\mathrm{RC}$ in the proposed method, its execution time will be reduced compared to the $\mathrm{RC}$ algorithm case. As a result, by coupling RC and DC computations, the execution time of the proposed method is almost one third of the execution time of the original RC method. In contrast, the computational time of LS-DCT method increases with $N^{3}$. In this computation, we use a matrix form of $2 \mathrm{D}$ discrete Fourier transform [32]. Through the use of matrix form, the computational time of $2 \mathrm{D}$ cosine transform needs only $N^{3}$ multiplications.

\section{B. Experimental Results}

The proposed algorithm has also been tested experimentally on a $2 \mathrm{D}$ wrapped phase map that resulted from the analysis of a real fringe pattern taken from the experiment carried out by using a Mach-Zehnder interferometer. The purpose of this experiment is to measure the phase shift in candle flames [23]. In this experiment, the exposure time can not be set long

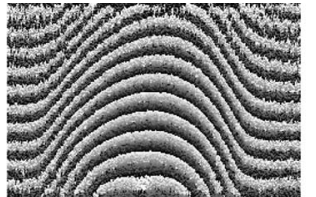

(a)

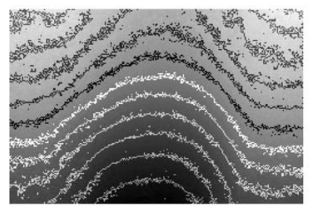

(c)

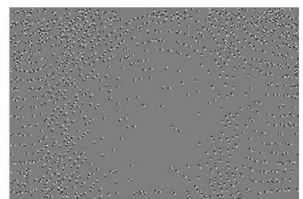

(b)

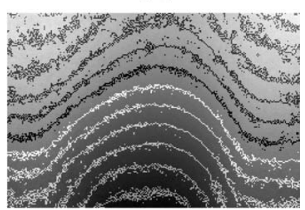

(d)
Fig. 5. Unwrapped phase result of experimental data for candle flame: (a) wrapped data, (b) SPs distribution map (positive and negative SPs are represented by white and black dots, respectively), (c) unwrapped result of the $\mathrm{RC}$ algorithm, and (d) unwrapped result of $\mathrm{RC}+\mathrm{DC}$ (proposed algorithm).

enough because the flame is varying in time by convection flow around the flame itself. Therefore, the fringe pattern has low signal-to-noise ratio; hence, it contains some defects. The phase data has image size 256 pixels $\times 170$ pixels and 2532 SPs (1267 positive SPs and 1265 negative SPs). The wrapped phase data and its corresponding SPs distribution map are shown in Figs. 5(a) and 5(b), respectively. Moreover, the unwrapped results, which have contour lines, obtained by RC method and the proposed algorithm are given in Figs. 5(c) and 5(d), respectively. By comparing the number of stripe lines in the wrapped phase data and the number of contour lines in the unwrapped results from the midpoint on the base line of each figure, it can be observed that the wrapped data has 10 lines, the unwrapped result of the RC algorithm has eight lines, and the proposed algorithm's result has nine lines. The unwrapped results in both methods are underestimated, however, the underestimation in the proposed algorithm is smaller than that in the RC method. This implies that the proposed algorithm succeeds to reduce the phase errors produced by the original RC method.

The execution time of the proposed algorithm varies depending on the number of SPs, data size, and the ratio of the adjoining SPs. Table 2 presents the execution time for simulated and real phase maps discussed above for each algorithm. The table shows the name, size, residue ratio, and adjoining SP ratio of each phase data. For the noisy phase map, the residue ratio, which is related to the data size, is around $9.1 \%$ and the adjoining SP ratio is $80.9 \%$. In this case, it was found that the overhead for each algorithm is the same. However, it is large enough compared to the execution time to compute the compensators for the proposed algorithm, i.e., $T_{\text {ovrh }}$ is almost 0.7 times $T_{\text {comp }}$, hence, the saving time cost ratio of the proposed algorithm is reduced from 77.6\% (saving time in compensators computation) to $67.2 \%$ (total saving time computation). On the other hand, flame data has residue ratio of approximately $5.8 \%$ and the adjoining SP ratio is around 
Table 2. Comparison of the Execution Time Cost between RC Algorithm and the Proposed Algorithm ${ }^{a}$

\begin{tabular}{|c|c|c|c|c|c|c|}
\hline Data name & $\begin{array}{l}\text { Data size: } \\
\quad N_{\text {all }}\end{array}$ & $\begin{array}{l}\text { SPs ratio: } \\
N_{s} / N_{\text {all }}(\%)\end{array}$ & $\begin{array}{c}\text { Adjoining } \\
\text { SPs ratio: } N_{a} / N_{s}(\%)\end{array}$ & $\begin{array}{c}\text { RC } \\
T_{\text {total }}(\mathrm{s}) \\
T_{\text {ovrh }}(\mathrm{s}) \\
T_{\text {comp }}(\mathrm{s})\end{array}$ & $\begin{array}{c}(\mathrm{RC}+\mathrm{DC}) \\
T_{\text {total }}(\mathrm{s}) \\
T_{\text {ovrh }}(\mathrm{s}) \\
T_{\text {comp }}(\mathrm{s})\end{array}$ & $\begin{array}{l}\text { Saving time } \\
\text { ratio }(\%)\end{array}$ \\
\hline Noisy phase & $100 \times 100$ & 9.1 & 80.9 & $\begin{array}{l}1.781 \\
0.240 \\
1.541\end{array}$ & $\begin{array}{l}0.585 \\
0.240 \\
0.345\end{array}$ & $\begin{array}{l}67.2 \\
77.6\end{array}$ \\
\hline
\end{tabular}

${ }^{a}$ The $T_{\text {ovrh }}$ presents the required execution time for overhead procedure to search and analyze the SP distribution. The $T_{\text {comp }}$ presents the required execution time to compute the compensators. The $T_{\text {total }}$ is the summation, $T_{\text {total }}=T_{\text {ovrh }}+T_{\text {comp }}$. $N_{s}$ and $N_{a}$, denote the total number of SPs and the number of SPs that form adjoining pairs, respectively. Saving time ratio $=1-T_{(\mathrm{RC}+\mathrm{DC})} / T_{\mathrm{RC}}$, where $T_{\mathrm{RC}}$ and $T_{\text {(RC+DC) }}$ represent the total execution times for the RC method and the proposed method, respectively.

$60.4 \%$. In this case, the overhead for the proposed algorithm is relatively small compared to the execution time to compute the compensators, i.e., $T_{\text {ovrh }}$ is almost 0.14 times $T_{\text {comp }}$, so that the saving time cost ratio of the proposed algorithm is reduced from $58.6 \%$ (saving time in compensators computation) to $55.5 \%$ (total saving time computation). However, the amount in reduction is not like the noisy phase case. In other words, the saving time ratio to compute compensators is almost the same to the adjoining SPs ratio. Therefore, the time to compute the DC is very small, so it is neglected and the saving time ratio to compute compensators is governed only by the RC computation.

From Table 2, it can be concluded that the execution time to search and analyze SPs is the same for the two examined algorithms. However, the execution time to compute the compensators in the proposed algorithm is reduced compared to that of the original RC method. This reduction in the required computation time of the proposed algorithm is due to the direct calculation of the compensators for the adjoining SPs pairs.

\section{Conclusion}

Several methods have been developed to solve phase unwrapping problems; nevertheless, providing satisfactory results leads to a time-consuming process. Phase unwrapping for noisy data by RC had higher accuracy than the other existing methods. However, it has a drawback of computational time requirement. To overcome this drawback, we propose a new method based on coupling the existing RC and the DC. The DC compensates the singularity of the pair of adjoining SPs connected by a branch cut with the length, which is shorter than 1 pixel. The compensator along the segment that crosses the branch cut is just $2 \pi$. For the SPs that are not members of adjoining pairs, $\mathrm{RC}$ is applied as a compensator. The proposed algorithm was tested on both computer-simulated and experimental noisy phase data. The results show that the proposed algorithm has a smaller computational time requirement compared to the original $\mathrm{RC}$ method, however, the execution time of the LS-DCT method is the least. Furthermore, the proposed method provides a more accurate unwrapped phase map than the past methods did.

\section{References}

1. D. C. Ghiglia and L. A. Romero, "Direct phase estimation from phase differences using fast elliptic partial differential equation solvers," Opt. Lett. 14, 1107-1109 (1989).

2. S. M. Song, S. Napel, N. J. Pelc, and G. H. Glover, "Phase unwrapping of MR phase images using Poisson equation," IEEE Trans. Image Process. 4, 667-676 (1995).

3. S. Chavez, Q.-S. Xiang, and L. An, "Understanding phase maps in MRI: a new cutline phase unwrapping method," IEEE Trans. Med. Imaging 21, 966-977 (2002).

4. R. M. Goldstein, H. A. Zebker, and C. L. Werner, "Satellite radar interferometry: two-dimensional phase unwrapping," Radio Sci. 23, 713-720 (1988).

5. I. Lyuboshenko and H. Maitre, "Phase unwrapping for interferometric synthetic aperture radar by use of Helmholtz equation eigenfunctions and the first green's identity," J. Opt. Soc. Am. A 16, 378-395 (1999).

6. M. Takeda, H. Ina, and S. Kobayashi, "Fourier-transform method of fringe-pattern analysis for computer-based topography and interferometry," J. Opt. Soc. Am. 72, 156-160 (1982).

7. K. E. Perry, Jr., and J. McKelvie, "A comparison of phase shifting and Fourier methods in the analysis of discontinuous fringe patterns," Opt. Lasers Eng. 19, 269-284 (1993).

8. E. Cuche, P. Marquet, and C. Depeursinge, "Spatial filtering for zero-order and twin-image elimination in digital off-axis holography," Appl. Opt. 39, 4070-4075 (2000).

9. J. H. Bruning, D. R. Herriott, J. E. Gallagher, D. P. Rosenfeld, A. D. White, and D. J. Brangaccio, "Digital wavefront measuring interferometer for testing optical surfaces and lenses," Appl. Opt. 13, 2693-2703 (1974).

10. B. Breuckmann and W. Thieme, "Computer-aided analysis of holographic interferograms using the phase-shift method," Appl. Opt. 24, 2145-2149 (1985).

11. J. M. Huntley, "Noise-immune phase unwrapping algorithm," Appl. Opt. 28, 3268-3270 (1989).

12. J. R. Buckland, J. M. Huntley, and S. R. E. Turner, "Unwrapping noisy phase maps by use of a minimum-cost-matching algorithm," Appl. Opt. 34, 5100-5108 (1995).

13. R. Cusack, J. M. Huntley, and H. T. Goldrein, "Improved noise-immune phase-unwrapping algorithm," Appl. Opt. 34, 781-789 (1995).

14. M. Costantine, "A novel phase unwrapping method based on network programming," IEEE Trans. Geosci. Remote Sens. 36, 813-821 (1998). 
15. B. Gutmann and H. Weber, "Phase unwrapping with the branch-cut method: clustering of discontinuity sources and reverse simulated annealing," Appl. Opt. 38, 5577-5593 (1999).

16. S. A. Karout, M. A. Gdeisat, D. R. Burton, and M. J. Lalor, "Two-dimensional phase unwrapping using a hybrid genetic algorithm," Appl. Opt. 46, 730-743 (2007).

17. D. L. Fried, "Least-square fitting a wave-front distortion estimate to an array of phase-difference measurements," J. Opt. Soc. Am. 67, 370-375 (1977).

18. R. H. Hudgin, "Wave-front reconstruction for compensated imaging," J. Opt. Soc. Am. 67, 375-378 (1977).

19. B. R. Hunt, "Matrix formulation of the reconstruction of phase values from phase differences," J. Opt. Soc. Am. 69, 393-399 (1979).

20. H. Takajo and T. Takahashi, "Least-squares phase estimation from the phase difference," J. Opt. Soc. Am. A 5, 416-425 (1988).

21. H. Takajo and T. Takahashi, "Noniterative method for obtaining the exact solution for the normal equation in least-squares phase estimation from the phase difference," J. Opt. Soc. Am. A 5, 1818-1827 (1988).

22. D. C. Ghiglia and L. A. Romero, "Robust two-dimensional weighted and unweighted phase unwrapping that uses fast transforms and iterative methods," J. Opt. Soc. Am. A 11, 107-117 (1994).

23. S. Tomioka, S. Heshmat, N. Miyamoto, and S. Nishiyama, "Phase unwrapping for noisy phase maps using rotational compensator with virtual singular points," Appl. Opt. 49, 4735-4745 (2010).
24. R. Yamaki and A. Hirose, "Singularity-spreading phase unwrapping," IEEE Trans. Geosci. Remote Sens. 45, 3240-3251 (2007).

25. C. L. Martinez and X. Fabergas, "Modeling and reduction of SAR interferometric phase noise in the wavelet domain," IEEE Trans. Geosci. Remote Sens. 40, 2553-2566 (2002).

26. M. R. Goldstein and C. L. Werner, "Radar interferogram filtering for geophysical applications," Geophys. Res. Lett. 25, 4035-4038 (1998).

27. J. S. Lee, K. P. Papathanassiou, T. L. Ainsworth, M. H. Grunes, and A. Reigber, "A new technique for noise filtering of SAR interferometric phase images," IEEE Trans. Geosci. Remote Sens. 36, 1456-1465 (1998).

28. C. L. Martinez, X. F. Canovas, and M. Chandra, "SAR interferometric phase noise reduction using wavelet transform," Electron. Lett. 37, 649-651 (2001).

29. Q. Kemao, S. H. Soon, and A. Asundi, "A simple phase unwrapping approach based on filtering by windowed fourier transform," Opt. Laser Technol. 37, 458-462 (2005).

30. Q. Kemao, "Two-dimensional windowed Fourier transform for fringe pattern analysis: principles, applications and implementations," Opt. Lasers Eng. 45, 304-317 (2007).

31. Q. Kemao, W. Gao, and H. Wang, "Windowed Fourier-filtered and quality-guided phase-unwrapping algorithm," Appl. Opt. 47, 5408-5428 (2008).

32. W. H. Press, S. A. Teukolsky, W. T. Vetterling, and B. P. Flannery, Numerical Recipes: The Art of Scientific Computing, 3rd edition (Cambridge University, 2007). 